\title{
ENVEJECIMIENTO MITOCONDRIAL
}

\section{La teoría del envejecimiento mitocondrial por la acción continua de los radicales libres del oxígeno y del nitrógeno}

\author{
Alberto Boveris ${ }^{1}$, Lidia E.. Costa ${ }^{2}$ y Virginia B.C. Junqueira ${ }^{3}$ \\ ${ }^{1}$ Instituto de Biología de los Radicales Libres, Facultad de Farmacia y Bioquímica, \\ ${ }^{2}$ Instituto de lnvestigaciones Cardiológicas, Facultad de Medicina, Universidad de \\ Buenos Aires y ${ }^{3}$ Departamento de Gerontología, Facultad de Medicina, Universidad \\ Federal de Sao Paulo.
}

\section{La función mitocondrial.}

La energía necesaria para las actividades celulares, tales como la biosíntesis, el transporte de iones y moléculas y el trabajo mecánico, proviene del ATP. En los mamíferos, aproximadamente el $95 \%$ del ATP se genera en las mitocondrias. En estas organelas subcelulares, presentes en todas las células aeróbicas eucariotes, el consumo de oxígeno está acoplado y provee la energía necesaria para la producción de ATP. Las mitocondrias dan cuenta de aproximadamente un 95\% del oxígeno consumido; entre 90 y $99 \%$ dependiendo del tipo celular y del estado metabólico. La energía del proceso por el cual los electrones se transfieren desde los substratos componentes del ciclo de Krebs hasta el oxígeno, está acoplada al transporte vectorial de $\mathrm{H}^{+}$desde la matriz mitocondrial al espacio citosólico (Fig. 1).

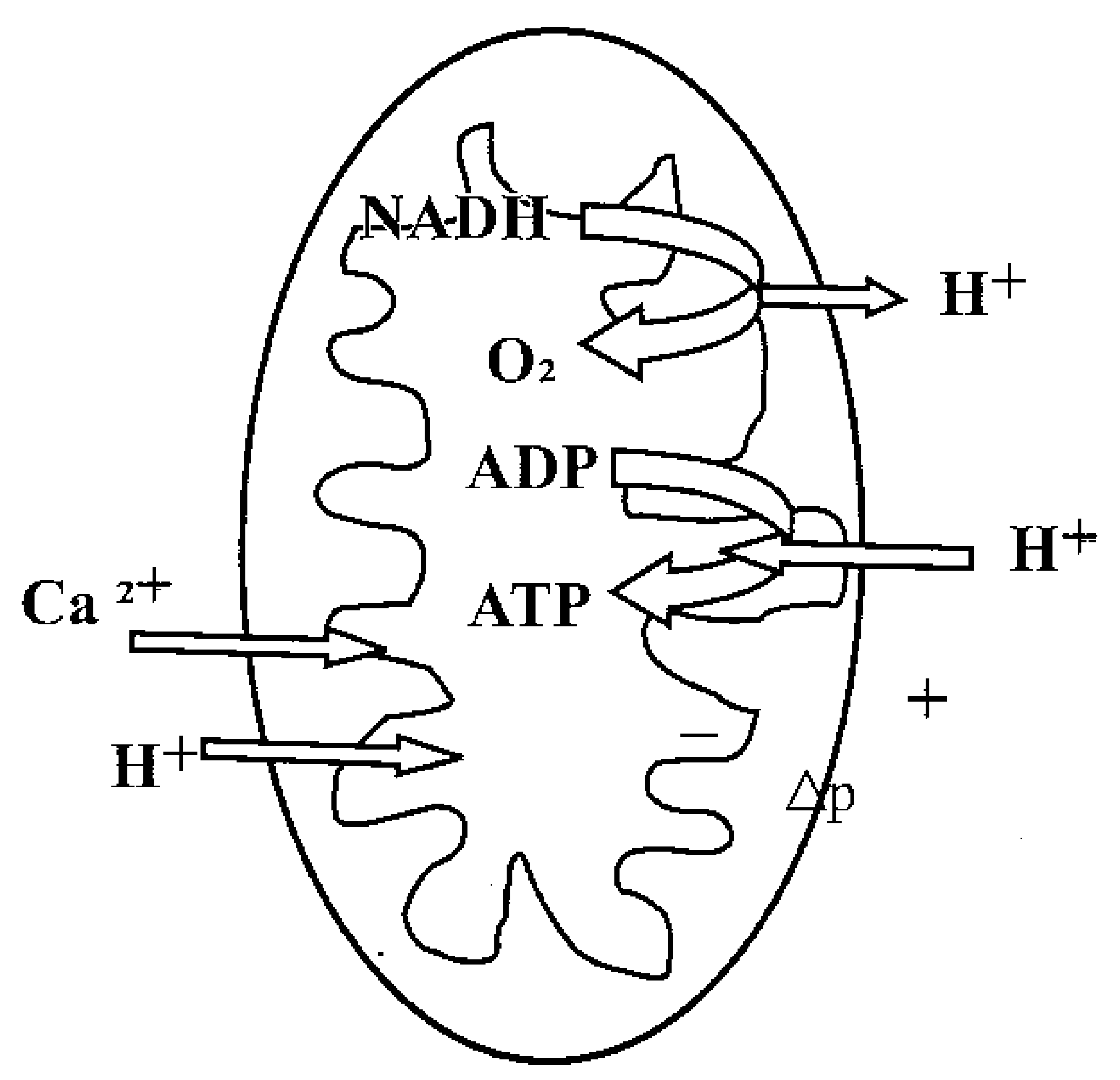

Fig. 1. La oxidación mitocondrial de los sustratos del ciclo de Krebs, representados por la coenzima reducida $\mathrm{NADH}$, se hace con la extrusión vectorial de $\mathrm{H}^{+}$hacia citosol generando un potencial protomotriz $(\triangle \mathrm{p})$ que dirige los $\mathrm{H}^{+}$de vuelta a la matriz mitocondrial a través de la $F_{1-}$ ATP asa que sintetiza ATP a partir de la energía libre del potencial electroquímico del $\mathrm{H}+$. Se indican la acumulación mitocondrial de $\mathrm{Ca}^{2+} \mathrm{y}$ la entrada pasiva de $\mathrm{H}^{+}\left(\mathrm{H}^{+}\right.$leak). 
El gradiente de $\mathrm{H}+$ a través de la membrana interna mitocondrial constituye una fuerza protomotriz $(\triangle p)$, que tiene dos componentes sumables $(\triangle p=\triangle p H+\triangle \Psi)$ que dan cuenta de la diferencia en la concentración de $\mathrm{H}^{+}(\triangle \mathrm{pH})$ y del potencial de la membrana $(\triangle \Psi)$, siendo este último el que contribuye con la mayor parte de la energía del gradiente.

El flujo de $H+$ de vuelta a la matriz mitocondrial, dirigido por el $\triangle \mathrm{p}$, se hace a través de la proteína $\mathrm{F}_{1}$-ATPasa, que actuando como un rotor molecular acoplado a los cambios de conformación de la macromolécula produce la síntesis de ATP a partir de ADP Y Pi. El potencial de la membrana interna mitocondrial es también utilizado para la homeostasis del $\mathrm{Ca}^{2+}$ citosólico que es acumulado en la matriz mitocondrial; el relativamente alto $\mathrm{pH}$ de la matriz mitocondrial estabiliza al $\mathrm{Ca}^{2+}$ que forma compuestos con ácidos orgánicos y con aniones fosfato, en este última caso formando la sal insoluble hidroxiapatita. El acoplamiento entre la extrusión de $\mathrm{H}^{+}$y la síntesis de ATP no es perfecto, debido a la perfecta impermeabilidad de la membrana interna mitocondrial a los $\mathrm{H}^{+}$; parte de éstos vuelven a la matrix mitocondrial sin pasar por la $F_{1}$-ATP asa y sin generar ATP en un proceso denominado "la entrada pasiva de $\mathrm{H}^{+}$" $\left(\mathrm{H}^{+}\right.$leak). Se ha propuesto que este proceso tiene un papel en la determinación de la velocidad del metabolismo basal.

\section{La producción mitocondrial de radicales libres del oxigeno y del nitrógeno.}

La transferencia de electrones desde los sustratos hasta el oxígeno a través de la cadena respiratoria mitocondrial tampoco es perfecta; una parte de los electrones reacciona directamente con el oxígeno molecular en un proceso denominado "la pérdida de $\mathrm{e}^{-"}$ ( $\mathrm{e}^{-}$leak) produciendo el radical libre anión superóxido $\left(\mathrm{O}_{2}{ }^{-}\right)$(Fig. 2).

Esta autooxidación de los componentes de la cadena respiratoria mitocondrial ocurre principalmente con (a) la ubisemiquinona, estabilizada por las proteínas de unión de ubiquinoma (QBP); y (b) la semiquinona de la flavoproteína de la NADH-deshidrogenasa. Las reacciones de autooxidación de la ubisemiqunona (reacción 1), conocida como reacción de Boveris-Cadenas (1976-1979), y de la NADH-deshidrogenasa (reacción 2), conocida como reacción de Boveris-Turrens (1979), dan cuenta, respectivamente, de un $75 \%$ y de un $25 \%$ de la producción mitocondrial de $\mathrm{O}_{2}{ }^{-}$.

Fig. 2. Producción mitocondrial de los radicales libres $\mathrm{O}_{2}{ }^{-}$y $\mathrm{NO}$. El radical $\mathrm{O}_{2}{ }^{-}$ es producido principalmente por la autooxidación de la ubisemiquinona (UQH.) unida a la proteína que se une a quinona (Q-binding protein, QBP), indicada en gris. La reacción de $\mathrm{O}_{2}^{-}$y $\mathrm{NO}$ (reacción de Padmaja-Beckman) genera $\mathrm{ONOO}^{-}$en la matriz mitocondrial. $\mathrm{El}_{2} \mathrm{O}_{2}$, producido por la dismutación de $\mathrm{O}_{2}{ }^{-}$, catalizada por la Mn-SOD, difunde de la matriz mitocondrial al citosol.

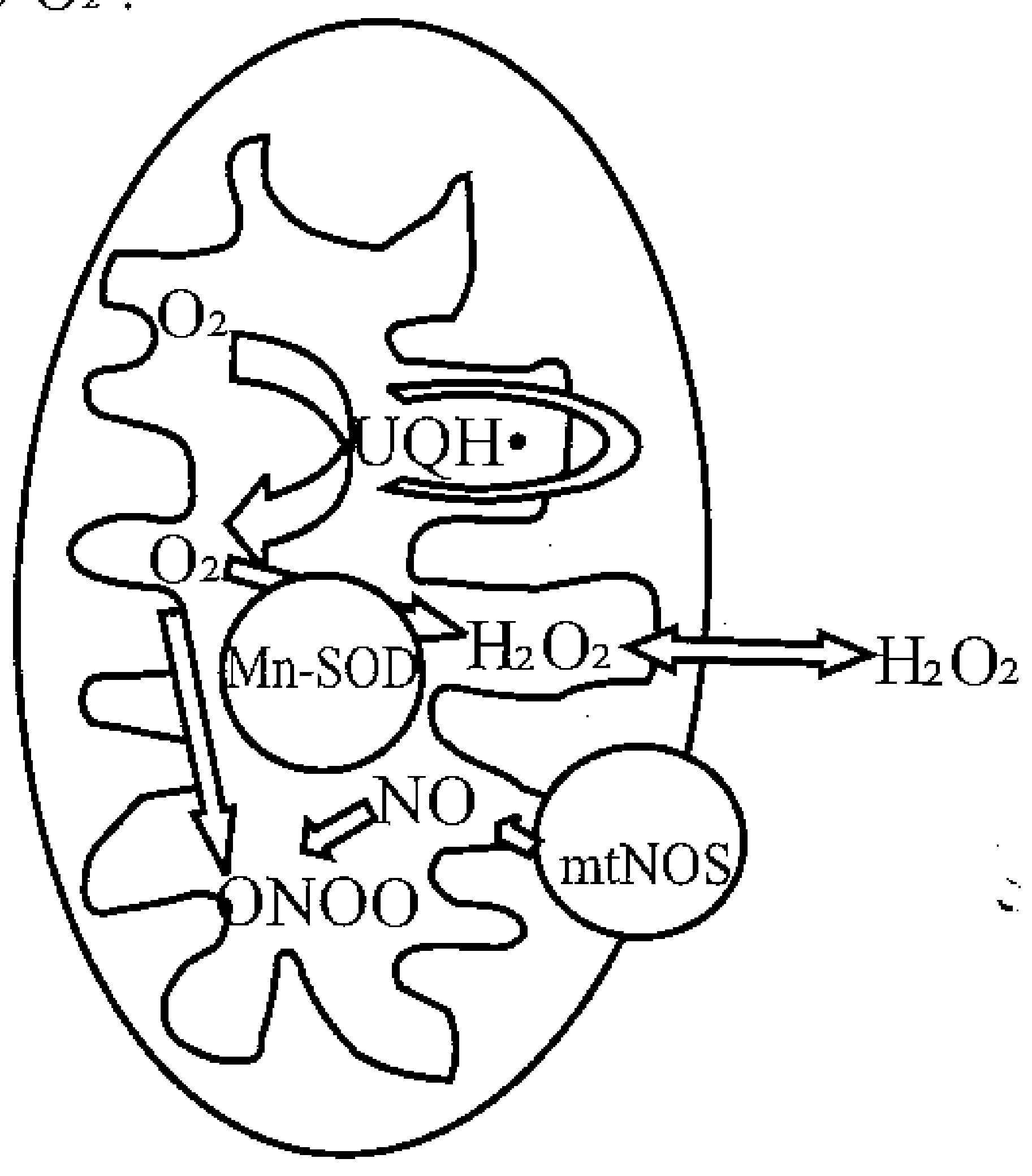


Se ha determinado que aproximadamente el $2 \%$ del oxígeno consumido en condiciones fisiológicas por. los órganos de los mamíferos es debido a la producción primaria de $\mathrm{O}_{2}{ }^{-}$. En el marco de la teoría del envecimiento mitocondrial por los radicales libres del oxígeno, la reacción de Boveris-Cadenas es considerada, en función de la producción continua de $\mathrm{O}_{2}{ }^{-}$, como el marcapaso del proceso de envejecimiento.

$$
\begin{array}{ll}
\mathrm{UQH}+\mathrm{O}_{2} \Rightarrow \mathrm{UQ}+\mathrm{H}^{+}+\mathrm{O}_{2}^{-} & {[1]} \\
\text { FMNH }+\mathrm{O}_{2} \Rightarrow \mathrm{FMN}+\mathrm{H}^{+}+\mathrm{O}_{2}^{-} & {[2]}
\end{array}
$$

$\mathrm{El} \mathrm{O}_{2}^{-}$generado en las reacciones (1) y (2) es producido vectorialmente hacia la matriz mitocondrial, espacio donde queda confinado por la impermeabilidad de la membrana interna mitocondrial al anión $\mathrm{O}_{2}{ }^{-} \mathrm{La}$ matriz mitocondrial contiene una superóxido dismutasa especial, la $\mathrm{Mn}-\mathrm{SOD}$, distinta de la dismutasa citosólica (Cu-Zn$\mathrm{SOD})$, que mantiene concentraciones en estado estacionario relativamente bajas $(0.1 \mathrm{nM})$ de $\mathrm{O}_{2}{ }^{-}$en el espacio intramitocondrial (reacción 3)

$$
2 \mathrm{O}_{2}^{-}+2 \mathrm{H}^{+}(\mathrm{Mn}-\mathrm{SOD}) \Rightarrow(\mathrm{Mn}-\mathrm{SOD}) \mathrm{H}_{2} \mathrm{O}_{2}+\mathrm{O}_{2} \quad \text { [3] }
$$

Recientemente, en 1998, se ha reconocido que la membrana interna mitocondrial posee una óxido nítrico sintetasa (mtNOS) que produce óxido nítrico (NO), un radical libre de oxígeno y nitrógeno con un electrón no apareado (reacción 4). El NO es generado vectorialmente en la matriz mitocondrial a velocidades comparables a las de generación de $\mathrm{O}_{2}{ }^{-}$

$$
\begin{gathered}
\left.\mathrm{NADPH}+\mathrm{Arg}+\mathrm{O}_{2} \mathrm{mtNOS}\right) \Rightarrow(\mathrm{mtNOS}) \\
\mathrm{O}_{2}^{-}+\mathrm{NODP}
\end{gathered} \underset{\mathrm{ONOO}^{-}}{\mathrm{NO}}+\underset{[5]}{\mathrm{Cit}}+\mathrm{NO}
$$

Los dos radicales libres, $\mathrm{O}_{2}^{-}$y $\mathrm{NO}$, reaccionan en la matriz mitocondrial muy rápidamente $\left(\mathrm{k}=1.9 \times 10^{10} \mathrm{M}^{-1} \mathrm{~S}^{-1}\right)$ en la reacción de terminación conocida como la reacción de Beckman-Padmaja (reacción 5), dando el poderoso oxidante peroxinitrito (ONOO). Actualmente se considera que el $\mathrm{NO}$ producido por la mtNOS es capaz de inhibir la actividad de la citocromo oxidasa y modificar las $\mathrm{K}_{0.502}$ (las concentraciones de oxígeno que sustentan la semimáxima velocidad de respiración mitocondrial) en condiciones fisiológicas. La mayor parte, $80 \%$, del $\mathrm{O}_{2}{ }^{-}$producido se utiliza de acuerdo a la reacción (3) y un $20 \%$, de acuerdo a la reacción(5). En el caso del NO, la mayor parte, $75 \%$, es utilizada de acuerdo a la reacción (5), y el resto en reacciones de reducción del NO por ubiquinol, citocromo c y citocromo oxidasa.

\section{La cadena de reacciones bioquímicas de radicales libres oxidativos.}

Las reacciones de producción primaria de $\mathrm{O}_{2}^{-}$y de $\mathrm{NO}$ en las membranas mitocondriales constituyen dos reacciones de iniciación de la reacción en cadena de radicales libres oxidativos que ocurre en condiciones fisiológicas en los órganos de los mamíferos (Figs. 2 y 3). El radical $\mathrm{O}_{2}{ }^{-}$, producido en las reacciones (1) y (2) lleva a la formación de $\mathrm{H}_{2} \mathrm{O}_{2}$ (reacción 3) y ambos productos de la reducción parcial del oxígeno en presencia de $\mathrm{Fe}$ como catalizador producen el reactivo radical hidroxilo (HO.) en la reacción de Fenton/Haber-Weiss (Fig. 2 y reacción 6). En esta reacción, el $\mathrm{O}_{2}{ }^{-}$, producto 


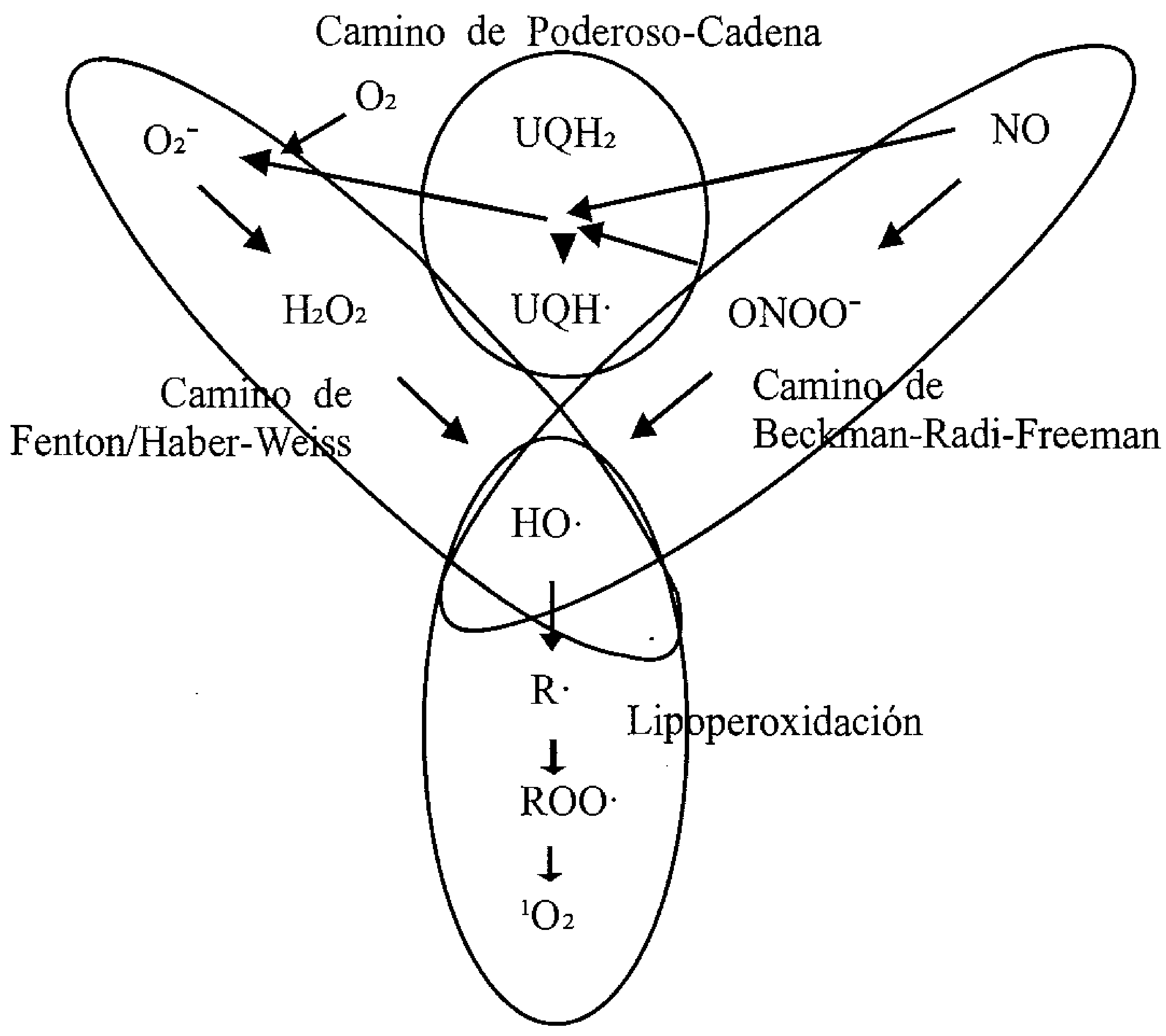

Fig. 3. Los caminos cruzados de la cadena de reacciones bioquimicas de radicales libres oxidativos. La producción primaria de los radicales libres $\mathrm{O}_{2}$ y $\mathrm{NO}$ en dos reacciones de iniciación, lleva en forma convergente a la generación del radical hidroxilo (HO) el que inicia las reacciones y el proceso de la lipoperoxidación.

de la reducción univalente del oxígeno, actuando secuencialmente genera al radical HO. Similarmente, la producción de $\mathrm{NO}$ (reacción 4) acoplada a la producción de $\mathrm{O}_{2}^{-}$y a través de la producción de $\mathrm{ONOO}^{-}$(reacción 5), lleva a través del camino químico de Beckman-Radi-Freeman (Fig. 2) y la reacción (7) a la producción de $\mathrm{O}_{2}{ }^{-}$

(a) $\mathrm{O}_{2}{ }^{-}+\mathrm{Fe}^{3+} \Rightarrow \mathrm{O}_{2}+\mathrm{Fe}^{2+}$; (b) $\mathrm{Fe}^{2+}+\mathrm{H}_{2} \mathrm{O}_{2} \Rightarrow \mathrm{Fe}^{3+}+\mathrm{OH}^{-}+\mathrm{HO} \cdot$ [6] $\mathrm{ONOO}^{-}+\mathrm{H}+\Rightarrow \mathrm{ONOOH} \Rightarrow\left[\mathrm{NO}_{2} \cdot \mathrm{OH}\right] \Rightarrow \mathrm{NO}_{2} \cdot+\mathrm{HO} \cdot \quad$ [7]

$\mathrm{El}$ radical $\mathrm{HO}$ es un oxidante extremadamente reactivo capaz de abstraer un átomo de hidrógeno de los carbonos alílicos $(=\mathrm{CH}-)$ de una multitud de constituyentes celulares, entre ellos los ácidos grasos insaturados de las membranas celulares y las bases púricas y pirimídicas de los ácidos nucleicos. Los compuestos resultantes de la 
abstracción de hidrógeno son radicales libres alquilo $(\mathrm{R} \cdot)$ (reacción 8 ). Los radicales libres de este tipo de las bases de los ácidos nucleicos frecuentemente se dimerizan, como es el caso de los dímeros de tímina. Los radicales alquilo derivados de los ácidos grasos insaturados son aptos para dar reacciones de adición con oxígeno molecular generando radicales peroxilo (ROO.) (reacción 9). los que a su vez, a través de una nueva abstracción de hidrógeno a otro carbono alílico de otro ácido graso insaturado, reacción favorecida por la estructura en bicapa lipída de las membranas biológicas, produce un hidroperóxido (ROOH) y un nuevo radical alquilo (R.) (reacción 10) lo que establece una reacción de radicales libres en cadena. En consecuencia las reacciones (8) a (10) constituyen reacciones de propagación clásicas, donde hay un radical libre entre los reactivos y un radical libre entre los productos.

$$
\begin{array}{cc}
\mathrm{HO} \cdot+\mathrm{RH} \Rightarrow \mathrm{R} \cdot+\mathrm{H}_{2} \mathrm{O} & {[8]} \\
\mathrm{R} \cdot+\mathrm{O}_{2} \Rightarrow \mathrm{ROO} \cdot & {[9]} \\
\mathrm{ROO} \cdot+\mathrm{RH} \Rightarrow \mathrm{ROOH}+\mathrm{R} \cdot & {[10]} \\
\mathrm{R} \cdot+\mathrm{ROO} \cdot(\mathrm{R} \cdot) \Rightarrow \mathrm{ROOR}(\mathrm{R}-\mathrm{R}) & {[11]} \\
2(\mathrm{ROO} \cdot) \Rightarrow[\mathrm{ROOOOR}] \Rightarrow \mathrm{ROH}+\mathrm{RO}+{ }^{1} \mathrm{O}_{2}[12]
\end{array}
$$

La peroxidación lípida o lipoperoxidación es el proceso químico donde las reacciones (8) a (10) ocurren sobre ácidos grasos insaturados. Los grupos - $\mathrm{OOH}$ de los hidroperóxidos $(\mathrm{ROOH})$, localizados en medio de la cadena hidrocarbonada de los ácidos grasos insaturados de las membranas celulares llevan a una distorsión del espacio hidrofóbico y a una pérdida de la función biológica de las membranas. La acumulación de grupos hidroperóxido en carbonos alílicos lleva a su vez a la ruptura de la cadena carbonada y a la formación de malonaldehído ( $\left.\mathrm{COH}_{-} \mathrm{CH}_{2} \mathrm{CHO}\right)$ que es un indicador de la ocurrencia de lipoperoxidación. Las reacciones (11) y (12) indican las reacciones de terminación que involucran a radicales alquilo y peroxilo. Cabe señalar que a su vez, la reacción (12) indica la formación de oxígeno singulete $\left({ }^{1} \mathrm{O}_{2}\right)$, el estado electrónico excitado del oxígeno molecular, como producto secundario y marcador del proceso de lipoperoxidación. El ${ }^{1} \mathrm{O}_{2}$ es quimioluminiscente $\left({ }^{1} \mathrm{O}_{2} \Rightarrow 2 \mathrm{O}_{2}+\mathrm{hv}(634\right.$ y $711 \mathrm{~nm})$ y la emisión espontánea de luz roja de órganos de mamíferos in situ se toma como una evidencia de que la lipoperoxidación ocurre en forma significativa en condiciones fisiológicas. Se ha calculado que el oxígeno consumido en el proceso de lipoperoxidación (reacción 9) da cuenta de aproximadamente un 2-3\% del consumo de oxígeno total de los órganos y los tejidos. El conjunto de las reacciones (1) a (11) constituye un proceso que se desarrolla continuamente en condiciones fisiológicas y que se denomina la cadena de reacciones bioquímicas de radicales libres oxidativos (Fig. 3).

\section{La hipótesis del envejecimiento por radicales libres.}

La importancia biológica de los radicales libres del oxígeno fue adelantada por: Gerschman en 1954, al reconocer que: (a) los radicales libres del oxígeno son el mecanismo molecular común de la toxicidad del oxígeno y de la radiación, (b) que un aumento de radicales libres oxidantes o una disminución de los antioxidantes, llevan 
igualmente a daño celular (situación conocida actualmente como estrés oxidativo), y (c) que la toxicidad del oxígeno es un fenómeno continuo. Harman en 1956, incorporó estos conceptos en una teoría general del envecimiento por radicales libres, en la que se considera que los radicales libres producen un daño acumulativo y al azar en las macromoléculas biológicas lo que lleva a una disminución de las funciones vitales y al envejecimiento. La teoría de Harman recibió fuerte apoyo experimental en el efecto de algunos antioxidantes en producir un alargamiento de la vida de roedores (Tabla 1) y en otras especies animales.

Simultáneamente, siguiendo un concepto de Szilard en el cual las perturbaciones microscópicas en moléculas informacionales llevan a disfunción macroscópica, Orgel produjo en 1963 la explicación del envejecimiento como originado en la acumulación de errores accidentales de traducción del mensaje genético, los que alterando a los componentes responsables de la fidelidad del mismo mensaje genético, es decir al ADN y al ARN, aumentaba y multiplicaba la frecuencia de los errores de traducción, los que iban degradando, poco a poco e inexorablemente, la estructura de los organismos.

La concepción de Harman del daño oxidativo a macromoléculas, ha sido recientemente revisitada y refinada por Sohal, que expresa al envejecimiento como consecuencia de la acumulación selectiva de daños oxidativos en algunas moléculas especialmente sensibles.

Tabla I. EFECTO DE ANTIOXIDANTES EN LA LONGITUD DE LA VIDA DE RATONES. La suplementación está indicada como g/\% en la dieta sólida. LAFI, C3H y SHK son cepas de ratones, $m$ y $h$, indican ratones machos o hembras.

\begin{tabular}{|c|c|c|c|c|}
\hline \multirow[b]{2}{*}{ Antioxidante } & \multicolumn{4}{|c|}{ Porciento de aumento en la longitud de la vida } \\
\hline & LAF1-m & $\mathrm{C} 3 \mathrm{H}-\mathrm{m}$ & $\mathrm{C} 3 \mathrm{H}-\mathrm{h}$ & SHK-f \\
\hline Mercaptoetilamina, $(0.5 \%)$ & 12.8 & & & \\
\hline Mercaptoetilamina $(1 \%)$ & 29.2 & & & \\
\hline Santoquina $(0.5 \%)$ & & 18.1 & 20.0 & \\
\hline 2-etil-6-metil-3hidroxipiridina & $(0.1 \%)$ & & & 23.1 \\
\hline Tomado de Harman (1982) & & & & \\
\hline
\end{tabular}


La hipótesis del envejecimiento por radicales libres se fortalece al focalizarla en las mitocondrias y se transforma en la teoría del envejecimiento mitocondrial y celular por la acción continua de los radicales libres del oxígeno y del nitrógeno. Las evidencias y los conceptos en apoyo de esta teoría son los siguientes: (1) Las mitocondrias son los sitios de producción de la energía celular (ATP) y una disfunción mitocondrial lleva a un estado de baja disponibilidad energética afectando todo el metabolismo celular. (2) Las mitocondrias son los sitios subcelulares más importantes de generación de $\mathrm{O}_{2}{ }^{-}$en los órganos de los mamíferos. Las mitocondrias también generan NO y estos dos radicales libres llevan a la formación intramitocondrial del radical $\mathrm{HO}$ y de $\mathrm{ONOO}^{-}$, dos poderosos oxidantes. La concentración intramitocondrial de $\mathrm{O}_{2}{ }^{-}$es de 5 a 10 veces mayor que la concentración de $\mathrm{O}_{2}{ }^{-}$en el citosol y en el núcleo. (3) $\mathrm{El} \mathrm{ADN}$ mitocondrial, localizado en la matriz de estas organelas, está muy cerca de los sitios de generación de $\mathrm{HO} \cdot$ y de $\mathrm{ONOO}^{-}$y no está protegido por las histonas ni tiene un efectivo sistema de reparación, como es en el caso del DNA nuclear. El DNA mitocondrial que se aisla normalmente tiene una cantidad de bases modificadas oxidativamente (por ejemplo. 8HO-deoxiguanosina) que es 10 a 20 veces mayor que la del DNA nuclear. (4) El DNA mitocondrial codifica aproximadamente un $5 \%$ de las proteínas de la cadena respiratoria. Los trece péptidos, componentes de la cadena respiratoria y de la $F_{1}$-ATPasa, sintetizados de acuerdo a la codificación del ADN mitocondrial son: (a) siete de la NADHdehidrogenasa; (b) tres de la citocromo oxidasa; (c) dos de la F1ATPasa; y (d) uno del citocromo b. Una síntesis defectuosa de estos péptidos produciría un deterioro del mecanismo molecular de transducción de energía. (5) El deterioro acumulativo del mecanismo molecular de transducción de energía mitocondrial modifica a las organelas a mitocondrias disfuncionales (Fig. 4). Estas mitocondrias disfuncionales, con capacidad disminuida de generación de ATP, y capacidad aumentada de producción de radicales libres entran en una situación de estrés oxidativo y producen señales al citosol, que llevan: (a) cuando las señales están en un nivel relativamente bajo, a la eliminación de las mitocondrias defectuosas mediante la digestión.
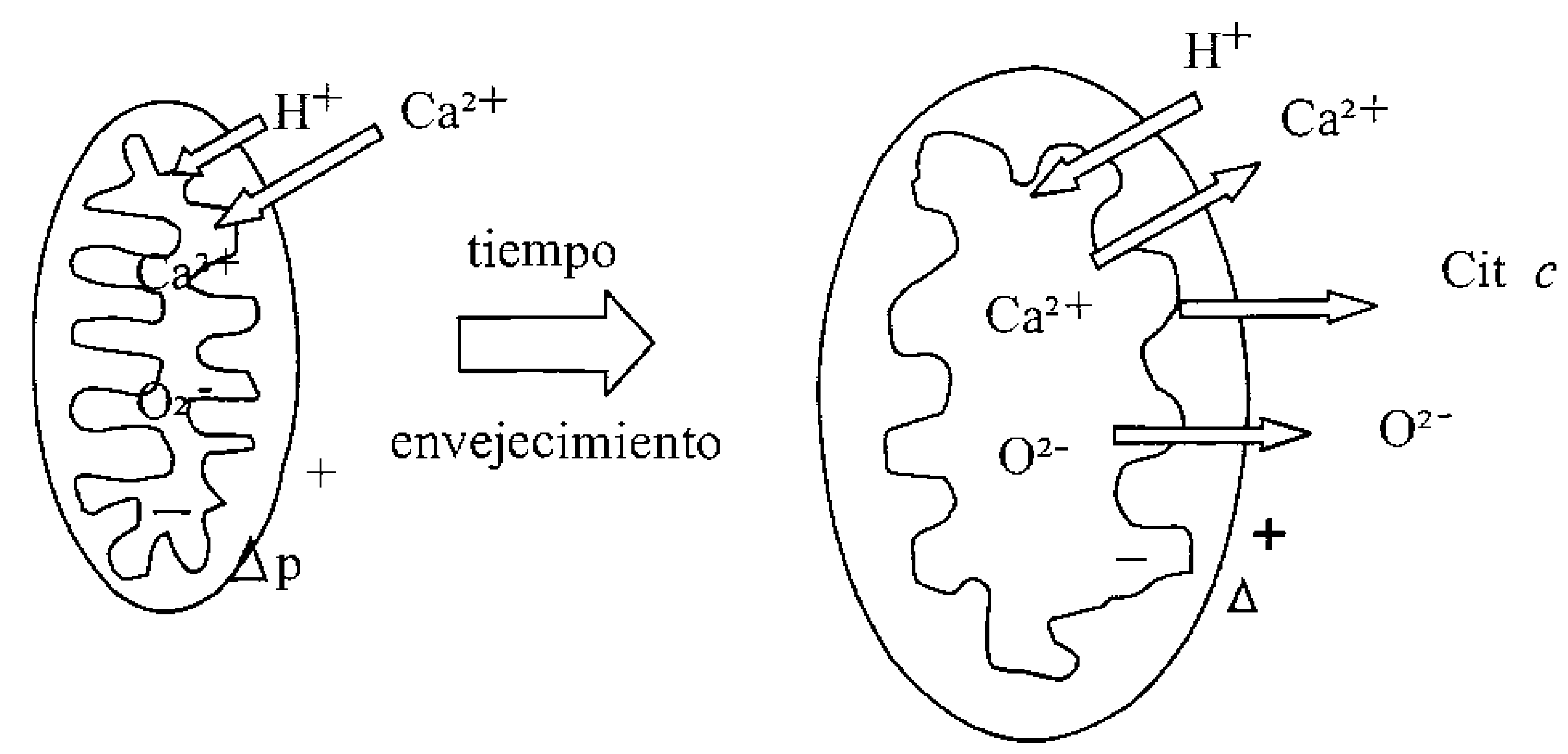

Fig. 4. La acumulación de daño oxidativo al DNA mitocondrial y a proteínas reguladoras de la función mitocondrial modifica a las mitocondrias normales (izquierda) en mitocondrias disfuncionales (derecha) en las cuales hay: (a) aumento de tamaño,(b) aumento de permeabilidad al $\mathrm{H}^{+}$con colapso del potencial protomotriz (hp), lo que lleva a un menor control respiratorio y a una menor síntesis de ATP, (c) salida de $\mathrm{Ca}^{2+}$ intramitocondrial, (d) liberación de $\mathrm{O}_{2}{ }^{-}$desde la matriz mitocondrial al citosol a favor del gradiente de concentración $\left(10^{-10} \mathrm{M}\right.$ en la matriz mitocondrial y. $10^{-11}$ en el citosol), y (e) liberación de citocromo $c$ desde la cara $\mathrm{C}$ de la membrana interna mitocondrial al citosol. 
lisosomal de las mitocondrias (hipótesis del eje mitocondrial-lisosomal en el envejecimiento celular), y (b) cuando las señales están en un nivel relativamente alto, lo que puede interpretarse como numerosas mitocondrias dando la señal de terminación, a la eliminación de la célula por apoptosis. La liberación del citocromo $c$ desde la cara esterna de la membrana interna mitocondrial al citosol es la señal intracelular más general y efectiva para la activación del proceso de muerte celular programada (Fig. 5). Las ideas acerca de la transición de mitocondrias normales a disfuncionales como consecuencia del paso del tiempo o del envejecimiento y la producción de scñales intracelulares desde las mitocondrias al citosol, tales como la liberación de $\mathrm{O}_{2}^{-}, \mathrm{Ca}^{2+}$ y citocromo $\mathrm{c}$, como señales de destrucción mitocondrial o celular constituyen las líneas de trabajo de los autores (Fig. 4 y 5).
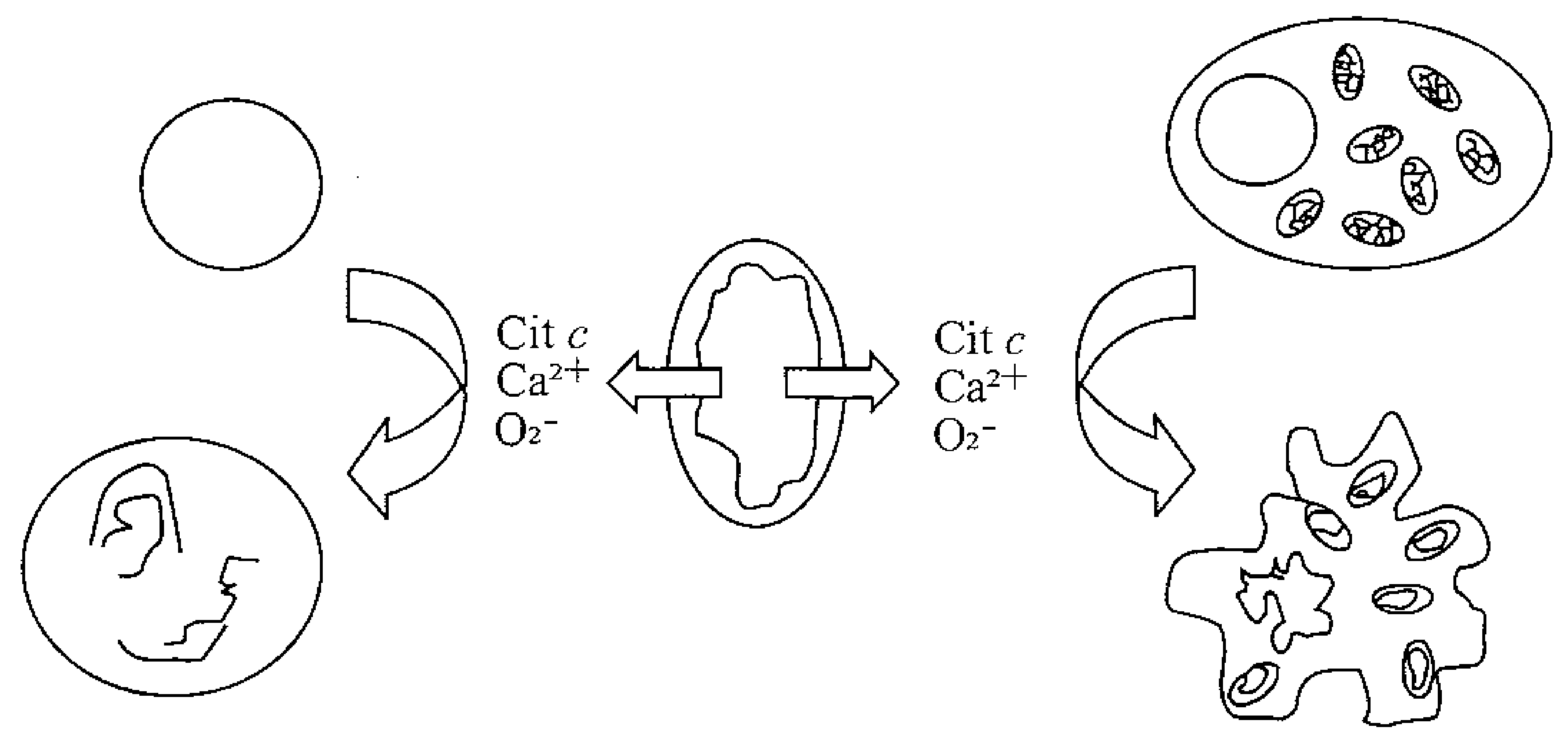

Fig. 5. Las señales mitocondriales de "desnutrición" $\left(\mathrm{O}_{2}{ }^{-}, \mathrm{Ca}^{2+}\right.$ y citocromo c) (a) (izquierda) en un nivel relativamente bajo, activan a los lisosomas primarios para la digestión de las mitocondrias, y (b) (derecha) en un nivel relativamente alto, activan los mecanismos celulares de muerte programada.

\section{La función mitocondrial en el envejecimiento.}

El punto central en la consideración de la teoría mitocondrial del envejecimiento es la obtención de evidencia mostrando que las mitocondrias de animales viejos tienen una disminución en la capacidad de producción de ATP. La cuestión parece trivial, pero se hace compleja al considerar que las mitocondrias disfuncionales pueden ser eliminadas por digestión lisosomal y que las células con mitocondrias disfuncionales pueden sufrir el proceso de apoptosis. En los órganos con capacidad dc regeneración, como el hígado, la proliferación celular puede ocultar casi totalmente la disfuncionalidad mitocondrial. Más aún, el proceso normal de aislamiento mitocondrial puede descartar casi totalmente a las mitocondrias disfuncionales con tamaño aumentando. Sin embargo, una serie de evidencias indican un daño selectivo en las mitocondrias durante el envejecimiento y la teoría del envejecimiento mitocondrial disfruta actualmente de reconocimiento y respeto.

Sohal ofrece la evidencia de que durante el proceso de envejecimiento en moscas domésticas hay una acumulación de carboxilación y una disminución de la actividad de la 
adeninanucleótido translocasa. Esta enzima de la membrana mitocondrial intercambia ADP/ATP entre la matriz mitocondrial y el citosol y tiene un papel esencial en la provisión de ATP a los procesos endergónicos de la célula que ocurren principalmente en el citosol.

La aconitasa, enzima soluble de la matriz mitocondrial que es parte del Ciclo de Krebs, también se ha encontrado carboxilada y con actividad disminuida durante el envejecimiento en moscas.

Desde la Universidad Complutense de Madrid, Barja y su grupo, y también Sohal han aportado abundante evidencia del papel de los radicales libres del oxígeno como determinantes de la longitud de la vida desde el enfoque de lá biología comparada. A través de una serie de especies, la longitud de la vida correlaciona negativamente con la velocidad de producción mitocondrial de radicales libres del oxígeno, a menor velocidad de producción mitocondrial de $\mathrm{O}_{2}{ }^{-}$mayor longitud de la vida.

En los últimos veinte años una serie de trabajos de microscopia electrónica ha informado una clara disminución de la fracción del citosol ocupada por las mitocondrias y del área de membrana interna mitocondrial durante el envejecimiento en hígado, corazón y cerebro de animales experimentales y de humanos. Las mitocondrias aparecen como las únicas organelas que muestran alteraciones morfológicas con la edad. Recientemente, Viña y su grupo en la Universidad de Valencia utilizando la citometría de flujo han reconocido dos poblaciones mitocondriales en el hígado y el cerebro de rata; una de mitocondrias pequeñas de escasa complejidad morfológica y la segunda de mitocondrias de gran tamaño y relativamente alta complejidad morfológica. Las mitondrias de gran tamaño y disfuncionales en cuanto a su potencial de membrana aumentan en proporción relativa con la edad. También, Navarro y su grupo en la Universidad de Cádiz han encontrado una fragilidad mitocondrial aumentada en higado y músculo de ratas durante el envejecimiento.

En las ratas viejas, se mide una actividad consịderable de complejos I, II, III y IV en el sobrenadante postmitocondrial del aislamiento de las mitocondrias. Las mitocondrias aisladas de animales viejos muestran frecuentemente, aunque no siempre, una disminución de la capacidad de transferencia de electrones por la cadena respiratoria. Las actividades de NADH-deshidrogenasa y de citocromo oxidasa, dos enzimas que contienen péptidos codificados por el DNA mitocondrial, se han encontrado disminuidas en corazón, músculo y cerebro de ratas envejecidas.

La respiración activa en presencia de ADP (estado 3), la máxima velocidad fisiológica de síntesis de ATP, se ha encontrado 45-50\% disminuida en ratones con senescencia acelerada. A su vez, mitocondrias aisladas de moscas y ratones envejecidos exhiben una respiración aumentada en ausencia de ADP (estado 4). Esta disfuncionalidad implica un aumento de la "entrada pasiva de $\mathrm{H}^{+}$" y eștá acompañada por un aumento en la velocidad de producción de $\mathrm{O}_{2}^{-}$y $\mathrm{H}_{2} \mathrm{O}_{2}$. Un interesante experimento se muestra en la Fig. 6, donde el control respiratorio, que es la relación entre las respiraciones en estado 3/estado 4, y a su vez, el índice más sensible para medir la funcionalidad mitocondrial, se encuentra disminuido con la edad en las mitocondrias de hígado de ratas que recibierón una dieta con bajo contenido de vitamina $E$, mientras que no se observan cambios con la edad en las ratas que recibieron una dieta con alto contenido de vitamina E. Esta evidencia indica un claro efecto beneficioso de la suplementación con antioxidantes en relación a la conservación de la función mitocondrial durante en envejecimiento. 
Además de los cambios referidos, se han encontrado aumentos en los contenidos de hidroperóxidos ( $\mathrm{ROOH}$ ), de malonaldehído, de glutatión oxidado y de 8-HOdeoxiguanosina en las mitocondrias aisladas de animales viejos en comparación con animales jóvenes. Se ha informado también de aumento de las deleciones en el DNA mitocondrial con el envejecimiento en animales experimentales y de un deterioro del diálogo entre núcleo y mitocondrias en fibroblastos humanos en función de la edad.

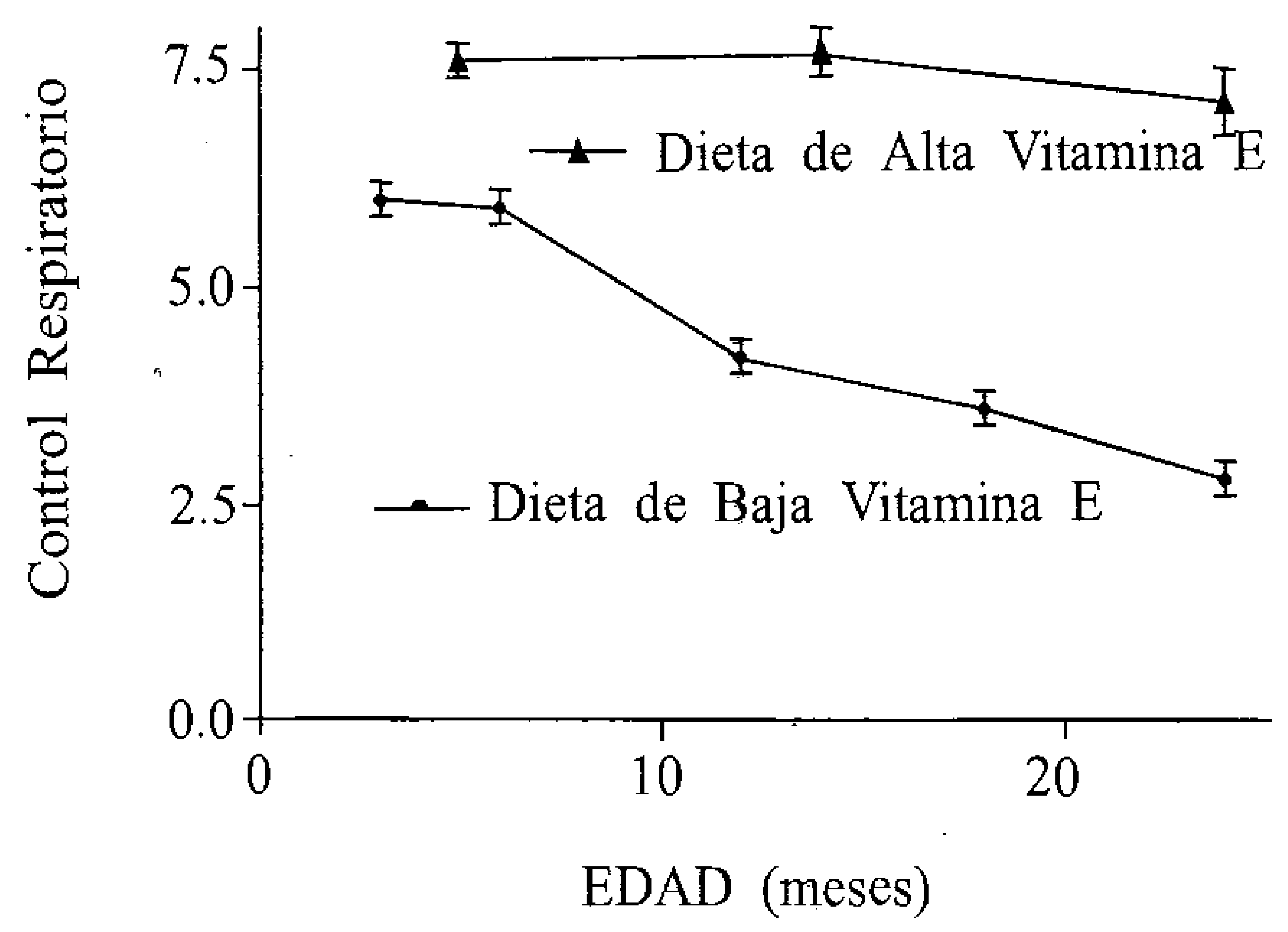

Fig. 6. Control respiratorio en mitocondrias de higado de rata en función de la edad. Sustrato: succinato. Contenido de vitamina $E$ de las dietas: alta vitamina $E$, $150 \mathrm{mg}$ de vitamina $\mathrm{E} / \mathrm{Kg}$ de dieta; dieta con baja vitamina $\mathrm{E}, 20 \mathrm{mg}$ de vitamina $\mathrm{E} / \mathrm{Kg}$ de dieta. Experimento de los autores.

\section{Conclusiones.}

En los últimos años se ha empezado a acumular evidencia experimental indicando que las mitocondrias evolucionan durante el envejecimiento a un estado de estrés oxidativo y disfuncionalidad. Los daños oxidativos ocurren selectivamente en algunos componentes de la cadena de transporte de electrones, específicamente en aquellos componentes cuyos péptidos constitutivos están codificados en el DNA mitocondrial. Paralelamente, se ha observado un incremento en la producción de radicales libres mitocondriales durante la disfuncionalidad mitocondrial; este estado parece llevar a través de señales en cascada a la digestión de las mitocondrias en lisosomas secundarios o a la apoptosis. En resumen, la teoría del envejecimiento mitocondrial por radicales libres, involucrando daños al DNA mitocondrial y señales de autodestrucción para las organelas y las células, ha alcanzado madurez y está lista para sugerir y promover nuevos experimentos extrapolables a todas las situaciones de proliferación/muerte celular, es decir a toda la biología celular. 


\section{REFERENCIAS Y LECTURAS RECOMENDADAS}

BOVERIS A, COSTA LE, CADENAS E. The mitochondrial production of oxygen radicals and cellular aging. En: "Understanding the process of aging. The roles of mitochondria, free radicals and antioxidants". (Eds.: Cadenas E, Packer L) Marcel Dekker, New York, (1999). pp. 1-16.

BOVER1S A, COSTA L.E., CADENAS E, PODEROSO JJ. Regulation of mitochondrial respiration by adenosine diphosphate, oxygen, and nitric oxide. Meth. Enzymol. 301: 188-198 (1999)

BOVERIS A. Biochemistry of free radicals: from electrons to tissues. Medicina 58: 350$356(1998)$

BOVERIS A., CADENAS E. Cellular sources and steady-state levels of reactive oxygen species. En: "Oxygen, Gene Expression and Cellular Function" (Eds.: Biadasz Clerch L, Massaro, DJ). Marcel Dekker, New York, 1997. pp. 1-25.

CHANGE B, SIES H, BOVERIS A. Hydroperoxide metabolism in mammalian organs. Physiol.Rev. 59: 527-605 (1979).

GIULIVI C., PODEROSO JJ, BOVER1S A. Production of nitric oxide by mitochondria. J. Biol. Chem. 273: 11038-11043 (1998).

HARMAN D. En "Free Radicals in Biology" (Ed. Pryor WA). Academic Press, Orlando. 1982. 255-275.

PEREZ CAMPO R, LOPEZ TORRES M, CADENASE., ROJAS C, BARJA G. The rate of free radical production as a determinant of the rate of aging: evidence from the comparative approach. J. Comp. Physiol. B. 168: 149-158 (1998).

VARIOS AUTORES en "Understanding the process of aging. The roles of mitochondria, free radicals and antioxidants". (ed. Cadenas, E., Packer, L.) Marcel Dekker, New York, (1999). 